\title{
Servicios amigables para jóvenes: Construcción conjunta entre jóvenes y funcionarios
}

\author{
Claudia Patricia Valencia, Enf, MSc ${ }^{1}$, Gladys Eugenia Canaval, Enf, MSc, PhD1, \\ Amanda Patricia Molina, Enf, MSc(c) ${ }^{2}$, Hugo Andrés $C_{\text {aicedo, }}$ NFF $^{2}$, \\ Lina Marcela Serrano, EnF ${ }^{3}$, Raquel Valencia, EnF $^{4}$, Vivian Piedrahita, EnF ${ }^{4}$,

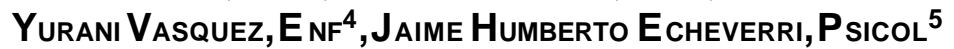

\section{RESUMEN}

Antecedentes: La atención integrada y amigable a los adolescentes en salud es un aspecto de vital importancia para el logro de indicadores en salud y para la prevención de comportamientos de riesgo que dejan consecuencias severas.

Objetivos: Identificar la percepción, experiencias y expectativas de jóvenes y funcionarios de salud sobre los servicios a jóvenes de la zona de ladera de Cali, Colombia. Desarrollar una estrategia conjunta entre jóvenes y funcionarios para el mejoramiento de los mismos servicios de salud a partir de los lineamientos de los Servicios Amigables para Jóvenes (SAJ).

Métodos: Estudio de investigación-acción-participación con jóvenes y funcionarios de una ESE de Cali. La población de jóvenes se encontraba entre los 10 a 19 años. Inicialmente se aplicó un instrumento de evaluación al personal de salud y luego una encuesta sobre los SAJ. Se e realizaron entrevistas grupales y grupos de discusión para analizar los resultados. Se hizo abogacía y se formularon propuestas ante las autoridades locales y tomadores de decisiones sobre el manejo de los recursos.

Resultados: La encuesta al personal de salud mostró que en la mayoría de las instituciones prestadoras de salud, no se cuenta con espacios y horarios definidos para la atención a los jóvenes, falta capacitación y número adecuado de funcionarios para la atención, ausencia de material de apoyo para educación en salud. La encuesta de jóvenes fue respondida por 100 jóvenes, el 76\% fueron mujeres. El promedio de edad fue de 15.7 años con un rango entre los 12 y 26 años; con relación a la satisfacción en la atención brindada por el personal de salud, el porcentaje más alto lo obtuvo el personal médico y el más bajo el personal auxiliar de enfermería. El servicio más consultado es la consulta con médico general y los servicios más frecuentados en el último mes fueron vacunación (19.7\%), odontología y urgencias (11.8\% para cada uno).

Conclusiones: Es necesario y de gran utilidad reorientar los servicios de salud que se ofrecen al joven de acuerdo a los principios y lineamientos de los SAJ, los cuales enfatizan en acciones de promoción de la demanda temprana y la prestación de servicios de atención integral y sin barreras, así como fortalecer conocimientos y habilidades específicas de los prestadores de servicios para que identifiquen y atiendan a jóvenes en forma idónea.

Palabras clave: Servicios amigables en salud; Calidad de atención; Adolescentes; Salud sexual; Salud reproductiva; Jóvenes; VIH-SIDA.

Health care services for young people: A joint construction among youth and health personnel

\section{SUMMARY}

Background: To accomplish health indicators among youth and prevent risks that leave severe consequences, it is important to offer integral health care.

Objectives: To identify perceptions, experiences and expectations about health services for youth and health care agents

1. Profesora Titular, Escuela de Enfermería, Facultad de Salud, Universidad del Valle, Cali, Colombia. e-mail: claudia.p.valencia@correounivalle.edu.corgladys.canaval@gmail.com

2. Profesional del componente Salud, Proyecto Colombia, Cali. e-mail: amanmoles@yahoo.com blacbirdes@yahoo.com

3. Profesional, Coordinadora Programa Joven, ESE Ladera, Cali, Colombia. e-mail: limarsnina@ gmail.com

4. Estudiantes en Trabajo de Grado, Escuela de Enfermería, Programa Enfermería, Universidad del Valle, Cali, Colombia. e-mail: selenedarien@hotmail.com raquelvalenciao@yahoo.es yuvao3645@hotmail.com

5. Coordinador General, Proyecto Colombia-Cali. e-mail: coordinación@ gmail.com

Recibido para publicación noviembre 13, 2008 Aceptado para publicación enero 12, 2010 
from the Zona de Ladera in the city of Cali. To develop a concerted strategy with youth and health care agents to improve health services under the «Friendly Youth Services» (SAJ abbreviation in Spanish) guide.

Methods: Action - Research with participation of healthcare personnel, youth, and adolescents from 10 to 19 years of age, was conducted in a public health-care institution in the city of Cali. The methodology included interviews, focal and discussion groups to analyze results and define steps to change services for adolescents. Advocacy and proposals about resource management were made to the local authorities and decision makers.

Results: The main findings were lack of time schedules for services to adolescents and the absence of educational materials for them. The study noted a lack of resources like equipment, well-trained personnel - especially with positive attitude toward adolescents, appropriate location for delivery of services, and community participation in health matters. The interview was answered by 100 young individuals, $76 \%$ were female, ranging from 12 to 26 years of age; the higher score about satisfaction was for physicians and the lowest was for nursing assistants. Respondents mainly seek physician attention. The service most frequently sought in the last three months was dentistry, vaccinations, and emergency attention $(19.7 \%$, and $11.8 \%$, respectively).

Conclusions: It is necessary and useful to reorient healthcare services for young individuals according to SAJ principles to emphasize health promotion, access to services, and skills and knowledge of health personnel to most appropriately care for the youth.

Keywords: Friendly health services; Quality assurance; Sexual health; Reproductive health; Adolescents; Youth; HIV-AIDS.

Durante la adolescencia, los jóvenes desarrollan la identidad que tendrán como adultos y avanzan en su madurez física y emocional. Si bien la adolescencia suele ser una época de buena salud, muchos adolescentes a menudo tienen menos información, menos experiencia y menos facilidad que los adultos para acceder a servicios de salud y particularmente servicios de planificación familiar y salud reproductiva ${ }^{1}$. Es posible que los adolescentes sientan hostilidad por parte de los adultos al tratar de obtener información y atención en salud sexual y reproductiva. Durante la adolescencia se podría presentar un mayor riesgo de contraer infecciones de transmisión sexual (ITS) y VIH, embarazos no deseados y otras consecuencias en la salud que podrían afectar su futuro y el de sus comunidades. Además, las desigualdades de género, en especial las diferencias de poder en las relaciones, pueden limitar su capacidad de usar anticonceptivos o de acudir a servicios de salud reproductiva.

La inversión en programas de educación, promoción, prevención y atención en salud sexual y reproductiva para adolescentes y jóvenes es muy inferior a la inversión que debe hacer el Estado en el tratamiento de las consecuencias que se presentan cuando la sexualidad, en esta población, se desarrolla y sigue comportamientos de riesgo que arrojan resultados indeseados como embarazo, parto pre-término, aborto provocado, infecciones de transmisión sexual, VIH/SIDA, madre solterismo, violencia sexual, prostitución y su asociación con otras situaciones que alteran el desarrollo de adolescentes y jóvenes como el uso de sustancias psicoactivas, depresión y suicidio ${ }^{2}$.

Durante 2005 a 2008 se realizó en el país el Proyecto Colombia Fondo Mundial (PCFM) $)^{3}$ con el apoyo del Banco Mundial, en 48 municipios. Uno de sus componentes se orientó a transformar los servicios de salud para jóvenes y adolescentes. El presente artículo muestra los resultados de este proceso de transformación de servicios amigables para jóvenes en la Empresa Social del Estado (ESE) Ladera de Cali.

Durante la ejecución del PCFM en el municipio de Cali se identificaron conocimientos, actitudes y comportamientos de riesgo en adquirir VIH/SIDA y otras infecciones de transmisión sexual (ITS) en jóvenes y adolescentes que residen en una de las zonas receptoras de población desplazada 4 como el inicio a temprana edad (14 años), de relaciones sexuales su práctica bajo el efecto de bebidas alcohólicas $(16 \%)$ y de sustancias psicoactivas (3\%), tener dos o más parejas simultáneamente $(25 \%)$ y el bajo uso del condón en las relaciones coitales $(36 \%)$.

Estos hallazgos indicaron que casi todos los jóvenes encuestados obtienen información de temas que se relacionan con la educación y la salud sexual por parte de los servicios de salud, lo que determina la necesidad de mejorar la orientación y calidad de estos servicios ofrecidos a los jóvenes en la población de Cali, específicamente en la zona de ladera.

Una de las actividades propuestas por el PCFM en Cali, fue implementar la estrategia de Servicios Amigables para Jóvenes (SAJ) en los servicios de salud a fin de mejorar la atención a este grupo poblacional. La 
Unidad Básica de Acción (UBA) del PCFM, en Cali, fue la Red de Salud de Ladera que hace parte de las 5 ESEs que prestan servicios de salud en la red pública del municipio, tiene a su cargo más de 37 IPS catalogadas así: 1 hospital, 6 instituciones que cumplen las características de centros de salud, 17 puestos de salud en el área urbana, 13 puestos de salud en el área rural, que cubren las comunas 1, 3, 17, 18, 19, 20 y el sector rural. Según las últimas proyecciones se calcula que Cali cuenta con 2’369,696 habitantes de ellos se estima que en ladera habitan alrededor de 659,000 de los cuales aproximadamente entre $36 \%$ y $40 \%$ son jóvenes entre 10 y 29 años.

La definición de (SAJ) recomendada por la Organización Panamericana de la Salud (OPS) y el Fondo de Población de las Naciones Unidas (UNFPA) es: «servicios donde las y los adolescentes y jóvenes encuentran oportunidades de salud cualquiera que sea la demanda, siendo agradables a los/as adolescentes y a sus familias por el vínculo que se establece entre los/as usuarios/as y el proveedor de salud y por la calidad de sus intervenciones» 5 .

La estrategia SAJ, es una iniciativa que promueve la transformación de servicios tradicionales de salud, en espacios que contribuyen al desarrollo de una salud sexual y reproductiva plena y saludable, además de satisfacer necesidades y expectativas de las-os jóvenes, con altos estándares de atención integral, oportuna, pertinente y equitativa ${ }^{5}$.Los servicios amigables constituyen unindicador de calidad que visibilizan los objetivos y metas de desarrollo institucional de cualquier entidad de salud, y responde a los estándares de calidad establecidos por el Ministerio Colombiano de la Protección Social. Los SAJ, figuran como una estrategia para mejorar la SSR en el marco de los objetivos del Plan Nacional de Salud Pública deColombia, según el Decreto 3039/20076 y estánincluidos como parte las estrategias de atención en las nuevas guías de promoción de la salud y prevención de enfermedades en salud pública en el modulo de guía para la detección temprana de alteraciones del desarrollo de jóvenes de 10 a 29 años ${ }^{7}$. Adicionalmente con esta estrategia se responde a algunos de los objetivos y metas del milenio, objetivo 5: Mejorar la salud materna cuya meta es reducir, entre 1990 y 2015, la mortalidad materna en tres cuartas partes; reducción del embarazo en la adolescencia cuya meta es lograr, para el año 2015, el acceso universal a la salud reproductiva y el objetivo 6: Combatir el VIH-SIDA, el paludismo y otras enfermedades ${ }^{8}$.

Con el PCFM-Cali se orientó el fortalecimiento de los servicios de salud ofrecidos a los jóvenes de la zona de Ladera de Cali, bajo los lineamientos del modelo de SAJ, a partir de un diagnóstico situacional con la participación de los jóvenes y funcionarios de salud. El propósito de este proyecto fue fortalecer los servicios de salud sexual y reproductiva enfocados en el modelo de SAJ. Los objetivos específicos fueron: 1) Identificar la percepción, experiencias y expectativas de los jóvenes y de los funcionarios de salud sobre los servicios a jóvenes de la zona de ladera de Cali y 2) Desarrollar una estrategia conjunta entre jóvenes y funcionarios para mejorar los mismos servicios de salud a partir de los lineamientos de los SAJ.

\section{MÉTODOS}

Se desarrolló una investigación tipo Acción-Participación por etapas. En la primera, se exploraron las percepciones, experiencias y expectativas de los funcionarios, acerca de los servicios de salud. Se evaluaron los componentes de un servicio amigable para adolescentes y jóvenes a partir de un instrumento adaptado del referido por Quintana e Hidalgo ${ }^{9}$ y por la Alianza CIDEMOS-PROINAPSA-UIS de Bucaramanga (ejecutora del Proyecto Colombia en Bucaramanga). Los componentes de un servicio amigable son ocho, están definidos en el anexo 5 del módulo de indicadores del documento de SAJ del Ministerio de la Protección Social $^{5}$ y son: 1. Características físicas del establecimiento de salud, 2. Características de los profesionales, 3. Características de los procedimientos y protocolos, 4 . Características de la atención propiamente dicha del joven, 5. Disponibilidad de servicios, 6. Percepción de los jóvenes acerca del servicio, 7. Compromiso de la participación juvenil y comunitaria, 8. Sistemas de evaluación y monitoreo. La encuesta a funcionarios se planeó aplicarla a la totalidad del personal encargado de atender la población del programa joven de las diferentes instituciones de la ESE ladera. Por último se aplicó a $77 \%$ (20 de 26) que correspondieron a los que se encontraban disponibles en el período de estudio. Los que no diligenciaron la encuesta estaban en vacaciones (4) o en licencia (2).

Luego se aplicó una encuesta para evaluar la percepción de los jóvenes acerca del servicio, a una 
muestra de 100 jóvenes escolarizados residentes en las diferentes comunas de la ESE ladera (Comunas 1, 18 y 20). Se calculó el tamaño de la muestra de los jóvenes de acuerdo con los lineamientos de los estudios descriptivos y los siguientes parámetros: Z: $1.96,95 \%$ de nivel de confianza, $\mathrm{p}=10 \%$ (\% de jóvenes que consultaron a los SAJ en el último año en la institución). La selección de los jóvenes se hizo a propósito, por la dificultad de saber qué jóvenes habían asistido antes a los servicios amigables y que pudieran emitir un concepto sobre los mismos. Por tanto, se decidió aplicar la encuesta a los que hubiesen sido atendidos en el período de recolección de datos obtenidos en el mismo servicio. La participación de los jóvenes fue anónima y voluntaria. El Comité de Ética de la ESE Ladera, aprobó el proyecto y avaló el consentimiento informado que se diseñó para los jóvenes y funcionarios del estudio.

Esta encuesta se construyó a partir de la encuesta del Programa Focus ${ }^{10}$ adaptada por PROINAPSA (Centro Colaborador de OMS/OPS en Promoción de la Salud y Promoción de la Salud Sexual y Reproductiva). Actualmente una encuesta similar aparece en el anexo 10 del documento de SAJ del Ministerio de la Protección Social 5 .

La encuesta se validó a través de una prueba piloto con los jóvenes del Grupo Juvenil «El Club» de la Comuna 1, que se encontraban en el Plan de Formación del PCFM en el barrio Terrón Colorado de Cali, y aportó recomendaciones para ajustar y obtener la versión final de la encuesta. Las encuestas se digitaron y procesaron con el programa estadístico EpiInfo versión 3.3.

En la última etapa del proyecto se socializó con jóvenes líderes participantes del PCFM los resultados de la encuesta, se brindó información sobre los objetivos y beneficios de Servicios en Salud Amigables para Jóvenes y se construyó conjuntamente la estrategia para mejorar los servicios de salud que se ofrecían a los jóvenes de la zona.

Una vez sensibilizados en la estrategia, se realizaron acciones conjuntas con ellos para adecuar un espacio físico acorde con sus gustos y necesidades y se sistematizaron las recomendaciones para las directivas de la institución conducentes a obtener su puesta en marcha de manera oficial.

Al mismo tiempo, funcionarios del Programa del Joven de la institución en mención y del Proyecto Colombia-Cali, efectuaron acciones de cabildeo y ges- tión ante los directivos de los entes territoriales, de los administradores y de los gerentes de servicios de salud, a fin de conseguir recursos y contratar un equipo humano interdisciplinario para la atención de los jóvenes; además se expusieron los motivos tendientes a generar voluntad política para el funcionamiento del servicio.

\section{RESULTADOS}

Encuesta a funcionarios. Las preguntas sobre las características de cada componente de los SAJ se calificaron entre 1 y 5 . La máxima calificación obtenida para tres componentes fue tres; la Gráfica 1 muestra la calificación global de cada componente.

Los resultados de la encuesta por componentes mostraron que en la mayoría de las instituciones prestadoras de salud, no se cuenta con espacios y horarios definidos para atender a los jóvenes, falta capacitación y número adecuado de funcionarios para la atención, ausencia de material de apoyo para educación en salud, según la edad del joven. El tiempo asignado para la consulta es mínimo, no hay información que divulgue los servicios para jóvenes; no existe una red estructurada entre servicios de salud para jóvenes y la comunidad; no hay procesos que integren a la comunidad con el sector salud y no existe un protocolo para la atención a jóvenes.

Encuesta a jóvenes y adolescentes. La encuesta respondida por 100 jóvenes que aceparon participar voluntariamente mostró que $76 \%$ eran mujeres. El promedio de edad fue 15.7 años con un rango entre 12 y 26 años. La mayoría de los/as jóvenes encuestados eran solteros $(93 \%)$ y ninguno de ellos-as refirió tener hijos. Llama la atención que el 7\% se declaró en unión libre.

Respecto a las respuestas por componentes se obtuvo lo siguiente:

Servicios consultados. El servicio más utilizado por los jóvenes es la consulta con médico general, como lo muestra el Cuadro 1 y los servicios mas frecuentados en el último mes fueron, vacunación (19.7\%), odontología y urgencias (11.8\% para cada uno); llama la atención que los programas que se relacionan con la salud sexual y reproductiva son poco frecuentados por los-as jóvenes.

Acceso y atención en el centro de salud. El $70 \%$ 
Encuesta a funcionarios sobre Servicios Amigables para Jóvenes $(n=20)$

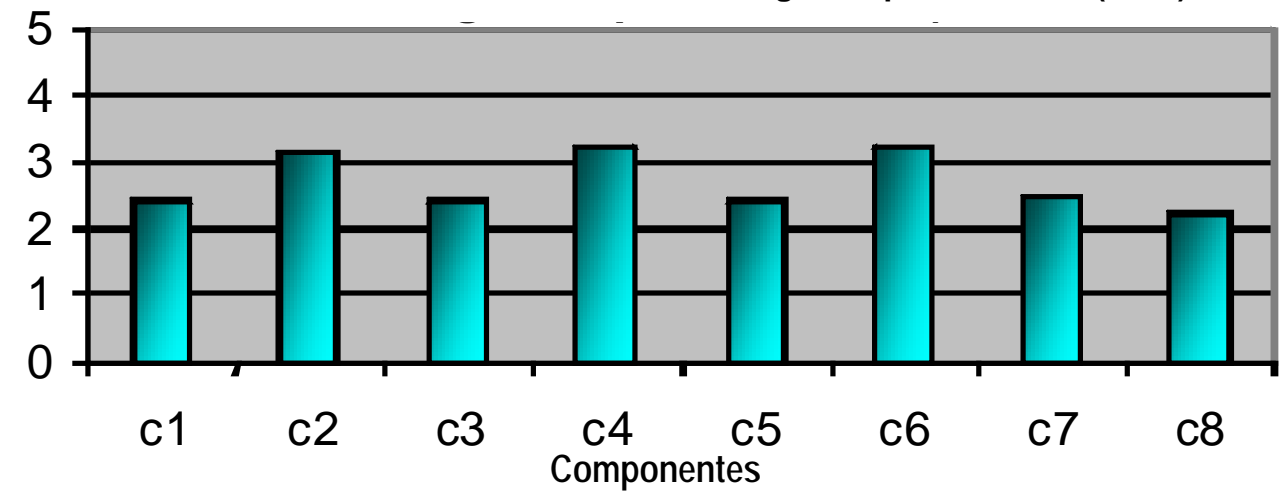

Gráfica 1. Calificación dada por funcionarios de salud, de los componentes de los servicios de salud ofrecidos a los jóvenes. Red de Salud Ladera 2007

Componentes: c1: planta física, c2: profesionales, c3: protocolos, c4: atención, c5: disponibilidad, c6: percepción de jóvenes, c7: participación juvenil, c8: monitoreo

Cuadro 1

Distribución de la frecuencia de uso, según tipo de servicio en la última visita realizada por jóvenes al servicio de salud. $n=76$

\begin{tabular}{|lc|}
\hline \multicolumn{1}{|l}{ Servicio de salud } & Porcentaje \\
\hline Odontología & 11.8 \\
\hline Consultamédicogeneral & 40.7 \\
\hline Planificaciónfamiliar & 7.8 \\
\hline Proyecto de jóvenes & 1.3 \\
\hline Citología & 3.9 \\
\hline Urgencias & 11.8 \\
\hline Psicología & 1.3 \\
\hline Prueba de VIH & 1.3 \\
\hline Vacunación & 19.7 \\
\hline
\end{tabular}

de los jóvenes consideró que es fácil llegar al centro de salud; el $34 \%$ refiere que no ha sido atendido en el horario en que se les había asignado la cita y el $62 \%$ refiere no haber sido atendido sin cita previa cuando lo necesitó. El $60 \%$ considera que durante la consulta se han utilizado los elementos necesarios y que el consultorio es amplio y privado pero no agradable; cerca de la mitad de los(as) jóvenes encuestados opinan que la atención en los servicios de salud es buena, aunque llama la atención que el 38\% no sabe cómo calificar la calidad de la atención. El material didáctico para refor- zar la educación en salud más utilizado son los volantes y folletos y los menos frecuentes pero los más llamativos para los jóvenes son los videos y la música.

Para la mayoría de los jóvenes (78\%) el tiempo destinado a la consulta fue suficiente y durante ésta lograron aclarar sus dudas. El 66\% tuvo la oportunidad de decidir si entraban acompañados o no a la consulta y $68 \%$ percibieron que se les trató como personas capaces de tomar sus propias decisiones. Sólo la tercera parte respondió que durante la consulta se habló acerca de su proyecto de vida y de sus derechos sexuales y reproductivos (Cuadro 2).

Es importante enfatizar que la mitad de los jóvenes refirió no haber recibido durante la consulta información de temas que les ofrezcan alternativas de prácticas seguras para el desarrollo pleno de su vida sexual; la información se centra especialmente en la enfermedad y su prevención (Cuadro 3).

En relación con la satisfacción con la atención brindada por el personal de salud, el porcentaje más alto lo obtiene el personal médico y el más bajo el personal auxiliar de enfermería. Hay que tener en cuenta que el porcentaje de satisfacción (sentirse bien) con el profesional de la salud no pasa en ningún caso de $80 \%$ (Cuadro 4).

Cabildeo, concertación y participación juvenil. Se concertaron varias actividades con los jóvenes para informarles acerca de la importancia de su participación activa y apropiación del servicio amigable. Se acordó 
Cuadro 2

Opinión de los jóvenes sobre la atención durante la consulta en salud

\begin{tabular}{lcccc|}
\hline Acerca de la consulta & N & Sí (\%) & No (\%) & No sabe (\%) \\
\hline El tiempo destinado para la consulta fue suficiente & 98 & 78.8 & 20.0 & 1.2 \\
\hline Escogiste entrar a la cita solo o acompañado & 98 & 66.3 & 25.5 & 8.2 \\
\hline $\begin{array}{l}\text { Te escucharon atentamente } \\
\text { Fuiste tratado como una persona capaz de tomar }\end{array}$ & 98 & 79.6 & 10.2 & 10.2 \\
tus propias decisiones & 98.0 & 68.4 & 13.3 & 18.4 \\
\hline $\begin{array}{l}\text { Conversaron acerca de tu proyecto de vida } \\
\text { Hablaron acerca de tus derechos sexuales }\end{array}$ & 97 & 31 & 59.8 & 9.3 \\
$\begin{array}{l}\text { y reproductivos } \\
\text { Incluyeron a tu pareja en la atención }\end{array}$ & 97 & 34.0 & 57.7 & 8.2 \\
\hline
\end{tabular}

\section{Cuadro 3}

Temas abordadas por los funcionarios de salud durante la consulta a jóvenes

\begin{tabular}{llccc}
\hline \multicolumn{1}{c}{ Temas } & N & Sí (\%) & No (\%) & No sabe (\%) \\
\hline Decisiones sobre comportamientos sexuales saludables & 87 & 41.4 & 49.4 & 9.2 \\
\hline Abstinencia y aplazamiento del inicio de la vida sexual & 80 & 35.0 & 51.3 & 13.8 \\
\hline Actividad sexual con protección para la sexualidad plena & 82 & 47.6 & 42.7 & 9.8 \\
\hline Uso del condón & 84 & 56 & 36.9 & 7.1 \\
\hline Métodos anticonceptivos, ventajas y desventajas & 78 & 51.3 & 41 & 7.7 \\
\hline Infecciones de transmisión sexual & 79 & 57 & 35.4 & 7.6 \\
\hline
\end{tabular}

Cuadro 4

Satisfacción de jóvenes con la atención recibida por el personal de salud

\begin{tabular}{lcccc}
\hline Personal de salud/Satisfacción con la atención & N & Bien (\%) & Regular (\%) & Mal (\%) \\
\hline Médico & 84 & 71.4 & 28.6 & - \\
\hline Enfermera(o) & 71 & 64.8 & 32.4 & 2.8 \\
\hline Psicólogo (a) & 58 & 58.6 & 29.3 & 12.1 \\
Aux. de enfermería & 64 & 45.3 & 45.3 & 9.4 \\
\hline
\end{tabular}

con las-os jóvenes varias reuniones de información para darles a conocer la propuesta del fortalecimiento del «Servicio de Salud para Jóvenes» y su horario amigable. Igualmente se les invitó a participar en la adecuación física del lugar de acuerdo con sus iniciativas (Figura 1). Se obtuvo autorización y apoyo de las directivas para la realización de un mural al estilo de los jóvenes. Se propuso un concurso para el diseño de un logo que identificara el servicio y se constituyera en un referente para los mismos jóvenes. Participaron cerca de 40 jóvenes de diferentes instituciones educativas de la Zona de Ladera y el jurado integrado por directivas, funcionarios del servicio de salud y jóvenes, escogió la propuesta que representó el sentimiento juvenil y la cultura caleña (Figura 2). 


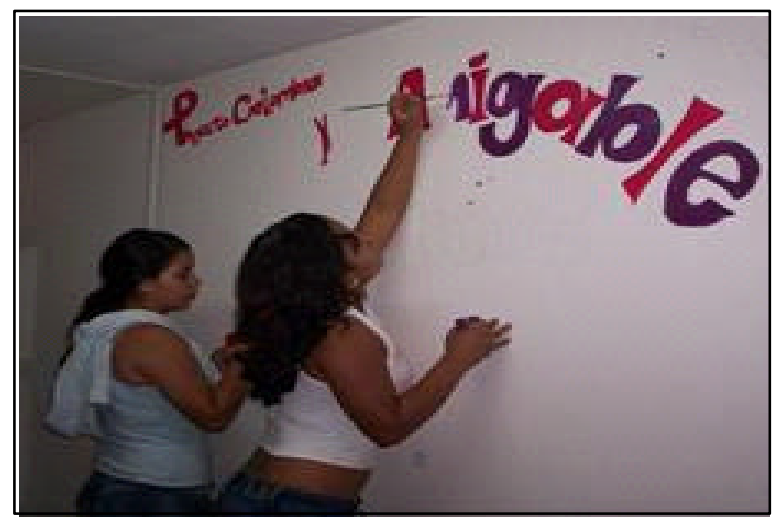

Figura 1. Jóvenes de la zona de ladera pintando un mural del consultorio para los SAJ

\section{DISCUSIÓN}

Los resultados de la encuesta aplicada a los funcionarios evidenció la necesidad de orientar un plan de mejoramiento en la línea de atención al joven en la institución de acuerdo con los lineamientos de los Servicios de Salud Amigables para Jóvenes, reglamentados para su implementación y cumplimiento en las IPS del país según el Ministerio de Protección Social de Colombia, Resolución 425 de $2008^{11}$. Esta resolución define la metodología para elaborar, ejecutar, seguir, evaluar y controlar las acciones de prevención de los riesgos en salud y de intervenciones colectivas (literal g del Artículo 17). Las áreas más críticas de los servicios de salud a los jóvenes, según el personal de salud consultado, fueron la ausencia de protocolos estandarizados para la atención del joven, la disponibilidad de servicios en horarios flexibles y la ausencia de indicadores y procesos de monitoreo.

Respecto a la percepción de los jóvenes y según la encuesta aplicada, las principales consultas a los servicios de salud (consulta médica, odontología y urgencias) son coherentes con las prácticas cotidianas de la población de acudir a los servicios de salud sólo cuando enfrentan un problema o enfermedad aguda, pues subvaloran o desconocen la utilidad de los servicios para las acciones de prevención y promoción de la salud. Llama la atención que los programas relacionados con la salud sexual y reproductiva son poco frecuentados por los-as jóvenes. Sin embargo, la asistencia frecuente de jóvenes y adolescentes a los servicios de odontología, vacunación y urgencias abre posibilidades de interven-

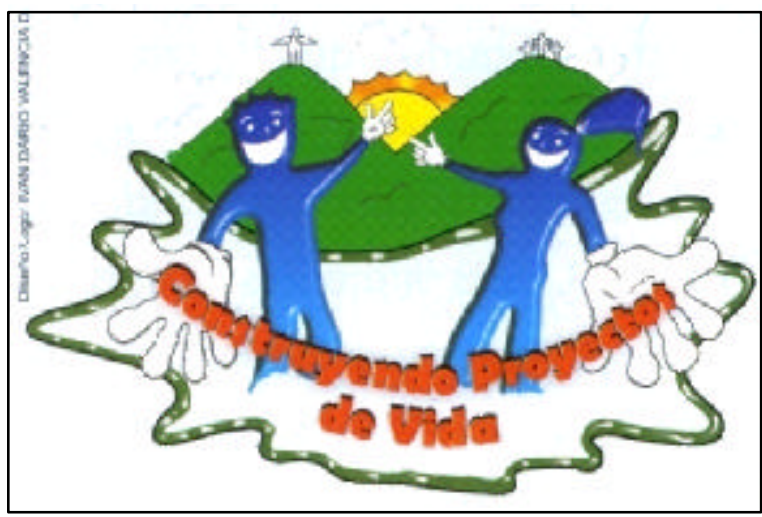

Figura 2. Logotipo ganador del concurso realizado por los SAJ, Zona Ladera, PCFM Cali, 2007

ción para captar jóvenes a través de la demanda inducida que pueden realizar las personas que trabajan en estas áreas, por lo cual se requiere ofrecer y mantener una capacitación para todo el personal de la institución que realiza actividades de atención al joven.

La mayoría de los enunciados valorados que obtuvieron puntajes adecuados entre los jóvenes, no representan a más de $60 \%$ de los encuestados. Sin embargo, para que el servicio sea amigable estos porcentajes deberían estar más cercanos al $100 \%$, como lo describen los estándares de la Iniciativa Nacional de Clínicas Amigables para Jóvenes (NAFCI siglas en inglés) ${ }^{12}$. En relación con la percepción de la calidad de atención, un gran porcentaje de los jóvenes, coinciden en afirmar que los servicios no son flexibles en el sentido que sólo se ofrecen con cita previa y los horarios no son de fácil acceso para ellos-as. Los servicios sin cita previa hacen que puedan sentir que cuentan con un horario amigable y que en el momento que requieran de algún tipo de asesoría o de la prestación de algún servicio se está en la disponibilidad para atenderlos.

Llama la atención que a $40 \%$ de los jóvenes encuestados no se les consultó sobre si deseaban entrar solos o acompañados a la consulta. Es de destacar que cada persona tiene derecho a elegir con quien entrar a la consulta, se debe salvaguardar la confidencialidad y la relación terapéutica que implica. Se hace necesario que esta actitud frente a la atención de los jóvenes sea distinta, porque un Servicio Amigable requiere que el personal establezca empatía con los jóvenes para que éstos no sientan que hay una barrera de comunicación 
y se motiven a regresar. Cabe anotar que el comienzo de la vida sexual implica un compromiso y responsabilidad compartida y es función del personal de salud que atiende a los-as jóvenes fomentar la participación en las decisiones que se tomen en SSR.

A pesar de la amplia difusión de los derechos sexuales y reproductivos y de ser considerados como una herramienta que permite a los jóvenes aumentar la capacidad para la toma de decisiones que aseguran su bienestar, llama la atención que menos del $60 \%$ de los encuestados dijo que este tema no se trató durante la consulta. De la misma forma, los resultados muestran que temas relacionados con la promoción de la salud sexual, como disfrutar de la sexualidad, el proyecto de vida, la toma de decisiones y algunos comportamientos de bajo riesgo, abstinencia y aplazamiento del inicio de la vida sexual, no se abordan en la consulta con la frecuencia que lo amerita. Esto tiene mayor relevancia si se reconoce que los jóvenes requieren más intervenciones que promuevan su salud y prevengan riesgos y enfermedades en virtud de las condiciones de salud, por lo general favorables, que presentan durante el ciclo vital que atraviesan. Estos temas los pueden abordar los miembros del equipo interdisciplinario con estrategias pedagógicas y metodologías para la educación en salud que sean llamativas a los jóvenes, lo que mejoraría sustancialmente la calificación dada a la satisfacción con la atención.

A pesar de la mayor participación de mujeres en la encuesta no se observaron diferencias por género en ninguna de las variables analizadas.

El $60 \%$ de los jóvenes, considera que durante la consulta se han utilizado los elementos necesarios y que el consultorio es amplio y privado, pero no resulta agradable para ellos, lo cual no les motiva su asistencia. La estética y el imaginario del adulto difieren de la del joven, por lo que se hace necesario comprometerlos en la adecuación de los espacios físicos, la promoción entre sus pares de los servicios y la apropiación de los mismos.

Las recomendaciones expresadas por los-as jóvenes para mejorar son las siguientes:

- Colocarse en el lugar de los jóvenes

- Usar animaciones más juveniles

- Sobre los funcionarios:

- Ser más amables, abiertos y alegres, prudentes, pacientes, tolerantes y atentos.

No ser groseros
Poner buena cara al atender al paciente

Que tengan siempre una sonrisa

Que no digan nombres extraños que no se entiendan Que proyecten confianza

De acuerdo con los resultados arrojados en la encuesta se hace evidente la necesidad de disponer de un lugar y un horario de uso exclusivo para la atención de los jóvenes, donde se sientan en confianza, cómodos y con privacidad. También se deben buscar lugares estratégicos de gran afluencia juvenil para garantizar la accesibilidad al servicio. Algunas experiencias han mostrado utilidad en la ubicación de establecimientos separados pero cercanos a los que los jóvenes podrían asistir sin encontrase con adultos que conocen, en los que se integran diferentes servicios a los jóvenes ${ }^{13}$.

El monitoreo de las acciones en el servicio de atención al joven en esta institución fue el de más baja calificación por parte de los funcionarios, lo que evidencia la inexistencia de indicadores para monitorear o el bajo análisis de los mismos.

El monitoreo y la evaluación constante son herramientas fundamentales que permiten obtener un panorama de los servicios de salud, mostrar debilidades y fortalezas y señalar puntos claves que permitan rediseñar las estrategias en la prestación de servicios. Una experiencia en Mozambique demostró que las áreas claves que contribuyen a tener éxito en la expansión de los programas de SSR y prevención de VIH entre jóvenes, incluyen el uso de datos de monitoreo y evaluación para guiar el cambio, la expansión y la flexibilización ${ }^{14}$.

Como lo refiere la literatura, los modelos de integración de servicios de SSR y del VIH para jóvenes son prometedores y pueden abordarse a partir de distintos enfoques: contar con un espacio para la atención, ofrecer servicios de divulgación para jóvenes en establecimientos existentes y hacer de estos sitios más agradables y amigables a los jóvenes ${ }^{15}$.

\section{CONCLUSIONES}

Los resultados de este trabajo evidencian la necesidad y utilidad de reorientar los servicios de salud al joven de acuerdo con los principios y lineamientos de los SAJ, que enfatizan en acciones de promoción de la demanda temprana y la prestación de servicios de atención integral y sin barreras, que prevengan los problemas que afectan a jóvenes y adolescentes con gran énfasis en el 
desarrollo del potencial humano y la calidad de la atención.

En consecuencia, es urgente desarrollar y fortalecer conocimientos y habilidades específicas de los prestadores de servicios para que identifiquen y atiendan a adolescentes y jóvenes en forma idónea, reconociéndolos como un grupo sano que requiere ser atendido de acuerdo con esta condición y destacar que sus motivos de consulta se relacionan principalmente con situaciones de la vida cotidiana y con la salud sexual y reproductiva.

Para que un servicio de salud sea amigable para losas jóvenes, es necesario evaluar constantemente la calidad en la atención a los usuarios y actualizar permanentemente al recurso humano, en temas de interés y en el trato adecuado con este grupo.

El interés permanente de directivos y funcionarios de la ESE Ladera en convertir los servicios de salud en servicios amigables para jóvenes permitió que este proceso se llevara a cabo, con los recursos existentes y posibilitó que la frecuencia de visita de jóvenes a los servicios de salud sexual aumentara; a su vez reorientó los servicios hacia la promoción de la salud y la prevención de la enfermedad, contribuyendo de esta forma a disminuir la problemática actual del país, en lo que respecta a las ITS/VIH/SIDA y a los embarazos no deseados, según lo planteado en las Metas del Milenio.

Conflicto de intereses. Los autores declaran que no hay conflicto de intereses en el presente manuscrito.

\section{REFERENCIAS}

1. Program for Appropriate Technology in Health (PATH). Adolescent reproductive health: making a difference. Outlook 16 (3) (December 1998). Disponible en: www.path.org/outlook/ html/16_3.htm\#Feat

2. Senderowitz J. Haciendo los servicios de salud reproductiva amigables para la juventud. FOCUS on Young Adults. Washington DC: Series de Investigación, Programas y Políticas; febrero 1999.

3. Proyecto Colombia. Construcción de una respuesta intersectorial para la disminución de la vulnerabilidad frente a las ITS y el VIH/SIDA en jóvenes en contexto de desplazamiento. Caja de herramientas. Instrucciones, Convenciones y Coordenadas. Módulo 1. Bogotá, DC: Fondo Mundial para la tuberculosis, el sida y la malaria; 2005.
4. Valencia CP, Canaval GE, Molina AP, Valencia R, Piedrahita $\mathrm{V}$, Vásquez Y.Conocimientos, actitudes y prácticas en salud sexual y reproductiva y prevención de ITS y VIH/SIDA de jóvenes en contexto de desplazamiento de Cali, 2007. Trabajo de Investigación de la Asignatura Trabajo de Grado, Escuela de Enfermería, Universidad del Valle, Cali; 2007.

5. Ministerio de la Protección Social \& Fondo de Población de las Naciones Unidas (UNFPA) Colombia.Servicios amigables en saludparaadolescentes yjóvenes. Unmodeloparaadecuarlas respuestas de los servicios de salud a las necesidades de adolescentes yjóvenes de Colombia. Bogotá,DC: TallerCreativo de Aleida Sánchez B. Ltda; 2007.

6. Ministerio de la Protección Social de Colombia. Decreto 3039/ 2007, por el cual se adopta el Plan Nacional de Salud Pública 2007-2010. Bogotá,DC: Ministerio de la Protección Social de Colombia; 2007.

7. Ministerio de la Protección Social de Colombia.Norma técnica para la detección temprana de las alteraciones del desarrollo del joven de 10 a 29 años. Bogotá, DC: Ministerio de la Protección Social de Colombia; 2007.

8. Organización de las Naciones Unidas. Objetivos de desarrollo del milenio. Ginebra: Organización de las Naciones Unidas; 2008.

9. Quintana A, Hidalgo C. Acortando distancias entre proveedores de salud y adolescentes y jóvenes. Servicios de salud amigables en Bolivia, Ecuador y Perú Lima: IES y Washington DC: Advocates For Youth; 2003. p. 88-91.

10. FOCUS on Young Adults. Servicios que dan buena acogida a los jóvenes. (octubre 10, 2008). URL disponible en: http:// www.fhi.org/sp/Youth/YouthNet/ProgramsAreas/Youth FriendlyServices/index.htm

11. Ministerio de Protección Social de Colombia.Resolución 425 de 2008. Bogotá, DC: Ministerio de Protección Social de Colombia.

12. Ashton J, Dickson K, Pleaner M.The evolution of the national adolescent friendly clinic initiative in South Africa. Geneva: WHO/CAH; 2007.

13. Dick B, Ferguson J, Chandra-Mouli V, Brabin L, Chatterjee S, Ross DA. Rewiev of the evidence for interventions to increase young people's use of the health services in developing countries. In: Ross D, Dick B, Ferguson J (eds.). Preventing HIV/AIDS in young people: a systemic rewiew of the evidence from developing countries. Geneva: World Health Organization; 2006. p. 151-204.

14. Hainsworth G. The Geracao Biz Programme in Mozambique, Draft. Geneva: WHO/Child and adolescent Health and Development ( $C A H)$ 2007. Presentación en la Interagency Youth Working Group Meeting, patrocinada por USAID, Washington, DC, May 2007.

15. Scholl $\mathrm{E}$, Finger W. Integración de servicios de salud reproductive y del VIH para jóvenes. Serie Lente Joven, Publicación $\mathrm{N}^{\circ} 21$. Arlington: Family Health International: 2008. 